\title{
A new GA based solution for capacitated multi source Weber problem
}

\author{
Nazila Mohammadi* \\ MSc Student, Faculty of Geodesy and Geomatics Engineering \\ K.N.Toosi University of Technology, Tehran,Iran \\ E-mail: nazila.mohamadi@gmail.com \\ Mohammad Reza Malek \\ Assistant Prof. Faculty of Geodesy and Geomatics Engineering \\ K.N.Toosi University of Technology, Tehran,Iran \\ E-mail: mrmalek@kntu.ac.ir \\ Ali Asghar Alesheikh \\ Associate Prof., Department of GIS Engineering, Geomatics Engineering Faculty, \\ K.N.Toosi University of Technology, Tehran,Iran \\ E-mail: alesheikh@kntu.ac.ir \\ Received: $27-04-2009$
Accepted: $25-06-2010$
}

\begin{abstract}
The main purpose of any facility location is to select the optimal places that satisfy project's goals. In location problems, the object is usually to optimize a function - objective function - that defines the problem conditions and efficient decision parameters. Numerous methods are proposed to challenge the location facility issues. In this paper, we consider a class of location/allocation problem that can assume more realistic conditions in real-life applications. This problem is an extension to the well-known capacitated multi source Weber problem. A new method that uses two genetic algorithms is used to solve the problem efficiently. The first, External GA, solves the location problem while the second, Internal GA, solves the allocation problem. A case study was designed to assess the feasibility of the proposed solution. The results indicate that the new approach is optimal, efficient and successful.
\end{abstract}

Keywords: facility location, optimization, objective function, location/allocation, GIS.

\section{Introduction}

Facility location is a branch of operations research concerning optimal placement of facilities. In a simple facility location problem a single facility is to be placed, subject to the minimization of the sum of distances from a given set of sites. More complex problems considers the placement of multiple facilities, constraints on the locations of numerous facilities, and various complex optimization criteria. ${ }^{1}$ Recent decades witness huge improvements in computational research and information technology and as such, the application of Geospatial Information Systems (GIS) in facility location is increased exponentially. ${ }^{2}$

Spatial scientists are continuously working on mathematical modeling and solution of problems concerning the placement of facilities to minimize transportation costs, prevent placing hazardous materials near housing, outrun competitors' facilities, etc. Bischoff et al. $^{3}$ formulated several objective

\footnotetext{
* Dept. of GIS, Faculty of Geodesy and Geomatics Eng., K.N. Toosi University of Technology, Department of GIS Engineering ValiAsr Street, Mirdamad cross, Tehran, Iran.
} 
functions in location problems. In the given formulation, all input values such as demand rate, distances and time of service transition are considered as fixed parameters, and then outputs are estimated as decision variables in one point of time. ${ }^{4}$

Location/Allocation (LA) problem is a class of facility location that has been studied for half a century because of its widely practical application backgrounds. The systematic study on location problem formally began by Weber in 1909 who considered how to position a single warehouse to minimize total distance between it and several customers. ${ }^{5}$ Location theory and its basic problems such as static and deterministic problems have been formulated since the mid 1960s. ${ }^{6}$ LA problem was studied in more detail in Ref. 7 and Ref. 8. Also in Ref. 9 and Ref. 10, you can see many models in this case.

Some of the problems require more than one facility centers to be located. Thus, multisource Weber problem (MSWP) is constructed. ${ }^{11}$ The multisource Weber problem is defined as locating simultaneously $\mathrm{m}$ facilities in the Euclidean plane in order to minimize the total transportation cost for satisfying the demand of $n$ users, each supplied from its closest facility. ${ }^{12}$

To solve such NP-hard (nondeterministic polynomialtime hard) problem, numerous methods have been proposed. ${ }^{13}$ Some of the solutions try to estimate local optimal or near optimal solutions of large problem instances. Other suggestions are made for heuristic as well as exact solution methods for the MSWP. Hansen et al. $^{14}$ solved the continuous location-allocation problem via the p-median problem by considering all points as potential facility sites. A genetic algorithm is designed by Houck et al. ${ }^{15}$ to solve a continuous location-allocation problem. A comparative study of local search methods such as variable neighborhood search, Tabu search and genetic algorithms has been carried out by Brimberg et al. ${ }^{16}$ Gamal and Salhi ${ }^{17}$ presented a constructive heuristic based on the longest distance rule to find initial locations while introducing forbidden regions to avoid locations being too close to each other. Salhi and Gamal ${ }^{18}$ adopted a genetic algorithm to solve such problems as well. Numerous randomized search methods were outlined for a generalized class of location/allocation problems by Bischoff and Dachert. ${ }^{19}$

A variant of location/allocation problem is the capacitated problem. In a capacitated problem, the customers may not be supplied by the nearest facility.
The earliest work in capacitated problem was conducted by Cooper $^{20}$ who developed exact and heuristic methods. Sherali and Rizzo ${ }^{21}$ studied the capacitated LA problem with a continuum of demand on a chain graph. Zainuddin and Salhi ${ }^{22}$ proposed a perturbation-based heuristic for the capacitated multisource Weber problem. Luis et al. ${ }^{21}$ put forward a new type of constructive and adaptive heuristics to generate initial solutions for the capacitated multisource Weber problem. This technique is based on guiding the search by constructing restricted regions that forbid new locations to be sited too close to the previously found locations. Doong et al. ${ }^{24}$ used a hybrid method of genetic algorithm and sub gradient technique to solve a class of capacitated facility location allocation problem. The main purpose of this paper is to present a method for solving the problem of Capacitated MSWP (CMSWP) class. We assumed new facility centers are to be located while considering existing centers. The proposed method uses two genetic algorithms in such a manner that one algorithm works inside another one. An external genetic algorithm chooses the locations for new facilities in each selection iteration, and an internal genetic algorithm determines the best allocation according to the newly chosen facility locations and existing facilities together. Finally, the result of these two genetic algorithms shows the best location and the best allocation for the entire region considering all new and existing facilities. In our approach, allocation varies based on the changes of locating new facilities. Therefore, optimum places constraining to the distances from customers as well as the newly introduced facilities are generated.

The paper is organized as follows. In section 2, the problem is formulated. Section 3 explains GA elements and the design of a powerful intelligent algorithm that includes two genetic algorithms. The results of a simulation are presented in section 4. Finally, we present concluding remarks in section 5.

\section{Problem Formulation}

In this study, we deal with a problem where we are given a set of customers, located at $n$ fixed points, with their respective demands. There are already $\mathrm{K}$ existing facilities in the region. We are required to locate $\mathrm{P}$ new facilities that these $\mathrm{P}+\mathrm{K}$ facilities serve all customers. Also we want to find the allocation of customers to the $\mathrm{P}+\mathrm{K}$ facilities while considering the capacity of each 
facility. The objective is to minimize the sum of the weighted Euclidean distances. There are R potential facility sites that we have to choose from them.

Choosing the best locations for establishing new schools can exemplify our problem. In such cases we can compose the close demand nodes and reach the demand district with the greater weight. We can do it, because usually neighbor pupils go to the same school.

To simplify the problem, we present solutions by some cells on a grid. In this problem, we use a regular tessellation of squared cells. The number of row and column is used to identify each cell location. Each cell has a demand weight according to the sum of the respective demand of customers referring to it.

For mathematical formulation of this problem, we added some terms to existing formulation of uncapacitated problem to cover the capacities. Therefore, decision variables defined as:

$$
\begin{aligned}
& X_{j}= \begin{cases}1 & \text { If a new facility locates at potential facility site } \mathrm{j} \\
0 & \text { Otherwise }\end{cases} \\
& Y_{i j}= \begin{cases}1 & \text { If demands at node } \mathrm{i} \text { are served by potential facility site at node } \mathrm{j} \\
0 & \text { Otherwise }\end{cases} \\
& Y_{i k}^{\prime}= \begin{cases}1 & \text { If demands at node } \mathrm{i} \text { are served by an existing facility at node k } \\
0 & \text { Otherwise }\end{cases}
\end{aligned}
$$

In the above equations $i$ is the index of a demand point, $\mathrm{j}$ is the index of a potential facility site and $k$ is the index of existing facility site. Accordingly, the problem can be formulated as (1) to (6).

$$
\begin{array}{lc}
\text { Minimize } \sum_{i}\left(\sum_{j} h_{i} Y_{i j} d_{i j}+\sum_{k} h_{i} Y_{i k}^{\prime} d_{i k}^{\prime}\right) \\
\sum_{j} X_{j}=p & \\
\sum_{j} Y_{i j}+\sum_{k} Y_{i k}^{\prime}=1 & \forall i \\
Y_{i j}-X_{j} \leq 0 & \forall i, j \\
\sum_{i} h_{i} Y_{i j} X_{j} \leq a_{j} & \forall j \\
\sum_{i} h_{i} Y_{i k}^{\prime} \leq a_{k} & \forall k \\
X_{j} \in\{0,1\} & \forall j \\
Y_{i j} \in\{0,1\} & \forall i, j \\
Y_{i k}^{\prime} \in\{0,1\} & \forall i, k
\end{array}
$$

Where $h_{i}$ is the demand at nod $i, d_{i j}$ expresses the distance between demand node $\mathrm{i}$ and the potential facility site $j$ and $d^{\prime}{ }_{i k}$ expresses the distance between demand node $\mathrm{i}$ and the existing facility $\mathrm{k}$. $\mathrm{p}$ is the number of facilities to be located. $a_{j}$ is the capacity of facility $j$ and $a_{k}$ is the capacity of existing facility $k$. As it can be seen in the above formula, the objective function (1) minimizes the total demand-weighted distance between facility centers and demand nodes. The constraints (2) control that exactly p new facilities be located. Eq. (3) ensures that all demands refer to centers. The family constraint (4) for new facilities allows assignment to sites which facilities have been located. Constraints (5) and (6) ensure that the capacity constraints of the facilities are not exceeded. The family constraints (7) to (9) are binary requirements for the problem variables. Considering the above nine formulas together, the approach leads to answer an optimization problem in which equation (1) is minimized while it satisfies constraints (2) to (9).

\section{The Proposed Method for Location- Allocation Problem}

In the proposed method, we use regular squares to divide the entire region into equal cells. Customers, facilities and potential facility places are defined through cells. Each cell has its own weight with respect to its costumer's demands.

In resent years, much attention is given to heuristic and search techniques. Genetic algorithms are also known as efficient heuristic and search techniques. ${ }^{25}$

Our proposed solution is mainly based on genetic algorithm. A GA search has the following advantages over traditional search methods: (i) GAs directly work with a coding of the parameter set; (ii) search is carried out from a population of points instead of a single one as in the case of the local search or simulated annealing algorithm; (iii) pay-off information is used instead of derivatives or auxiliary knowledge; and (iv) probabilistic transition rules are used instead of deterministic ones. ${ }^{24}$ GAs have been successfully applied to a diverse set of optimization problems. ${ }^{26}$ Our solution uses two genetic algorithms. One GA to solve the location problem that works as external algorithm. The other one solves allocation problem that works as internal genetic algorithm. 
Using such a method, we estimate the best location for new facilities, and the best allocation to all existing and new facilities.

\subsection{A review of $G A$}

This section is devoted to review the notation and concept of genetic algorithm (for more details see Ref. 27) that has been exercised in our methodology (Figure 1).

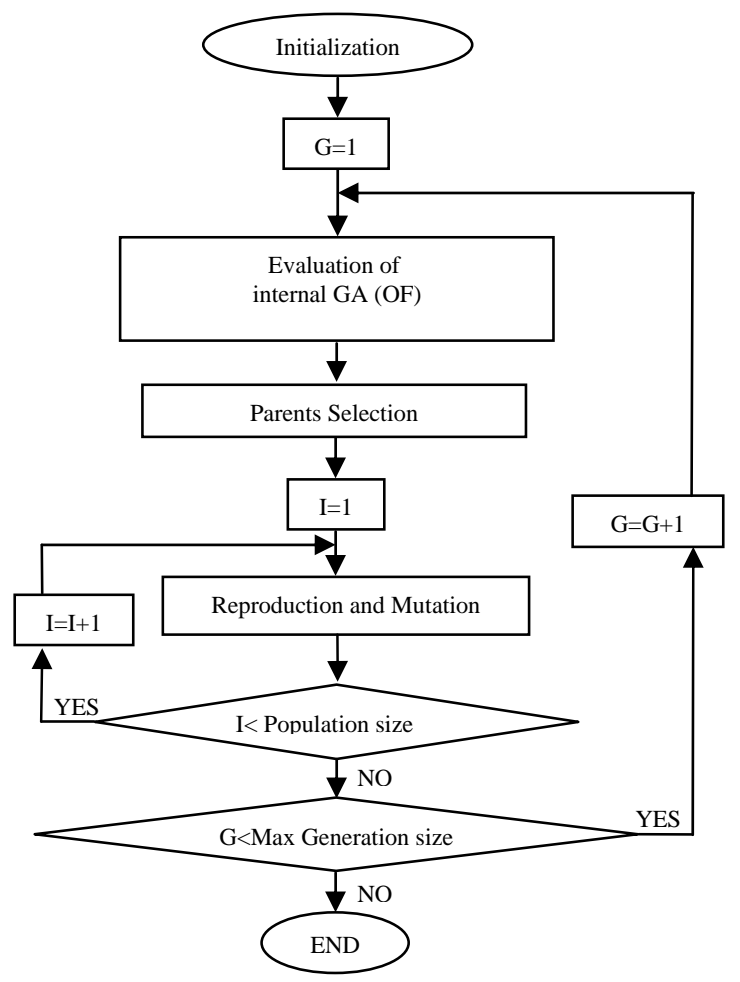

Fig. 1. Flow diagram of GA used in this research

Initialization:

The first "Parents" chromosome pool should be generated by randomly creating several sets of facility centers coordination such that all sets of the facility centers totally satisfy all the constraints. Each set of coordination setting is packed into a chromosome. The key variable in the GA is the chromosome.

Evaluation:

The OF (Objective Function) value which is shown in the flowchart and was described in section 2, is calculated to evaluate the effectiveness of the grading. The smaller the evaluated objective value is, the better the chromosome becomes.

\section{Selection:}

According to the calculated OF's values of "Parents" at each generation, some parents are more distinct than the others. Based on a concept called elitism, those parents who have more optimal OF values in the "Parents" chromosome pool, should be granted more opportunities to survive, so that they can generate more offsprings. The roulette wheel selection is used with this enhanced selection list.

Reproduction and mutation:

The reproduction is responsible for producing offsprings by crossover and mutation operators. Checking and evaluating of new offsprings is required to form the "Children" chromosome pool. The offsprings may be improved by making some favorable changes in crossovers and mutations. Mutation sometimes introduces new genetic material into a heterogeneous population; it may however introduce fault chromosomes. The probability of permutation should not be overemphasized. Moreover, all the mutated offsprings must be tested for constraint satisfaction and those that failed are eliminated.

\section{Chromosome selection for next Generation:}

It is common in optimization methods to find that the OF value is extremely sensitive to one parameter, whilst large changes are still needed in another parameter. GA is not an exception. To deal with such inequities, the pre-scaling technique is employed. The OF value of each chromosome of the "Parents" will be converted to a raw fitness, and then to a scaled fitness and finally to the expected frequency of selection as a parent.

Termination:

The process will be terminated after a fixed number of generations. Due to the fact that improvement may not appear quickly, more generations are preferable. This will improve the chromosome pool and will prepare better genetic materials for future generations. The required number of generations varies from one system to another. It depends on the system complexity and the population size.

\subsection{Adoption of GA}

This section presents the elements of each genetic algorithm that is used in the proposed methodology and indicates how to combine the GAs to obtain the best solution. 


\subsubsection{Internal $G A$}

Internal GA is the one that is applied to allocate demand cells with respect to their weights to $\mathrm{P}+\mathrm{K}$ facilities, where $P$ is the number of new facilities and $K$ is the number of existing facilities.

Each chromosome consists of $\mathrm{S}$ genes, where $\mathrm{S}$ is the number of cells. Assuming the whole region is covered by r rows and c columns, then $S=r^{*} c$.

Existing facility centers are coded from 1 to $\mathrm{K}$ and new facility centers are coded from $\mathrm{K}+1$ to $\mathrm{K}+\mathrm{P}$.

The genes of each chromosome can take one integer number from 1 to $\mathrm{P}+\mathrm{k}$. This integer number indicates a facility center that the demand cell is allocated to (Fig. 2). Therefore, all demand cells refer to facility centers.

\begin{tabular}{|l|l|l|l|l|l|l|l|l|}
\hline $\mathrm{g}_{1}$ & $\mathrm{~g}_{2}$ & $\mathrm{~g}_{3}$ & $\mathrm{~g}_{4}$ & & $\ldots$ & & $\mathrm{g}_{\mathrm{s}-1}$ & $\mathrm{~g}_{\mathrm{s}}$ \\
\hline
\end{tabular}

Fig. 2. The structure of a chromosome for inner GA

In this GA we assumed that the locations of new facilities are known and internal GA determines the best allocation of demand cells with $\mathrm{P}+\mathrm{K}$ centers.

Objective function for external genetic algorithm is defined by Eq. (10). Eq. (11) and (12) define the variables $\mathrm{t}$ and $\mathrm{q}$ and Eq. (13) defines the decision variable V. GA minimizes the following function.

$$
\begin{aligned}
& \text { OF }=E_{1} \sum_{i=1}^{S} h_{t, q} \sqrt{\left(r_{t}-r_{f_{g_{i}}}\right)^{2}+\left(c_{q}-c_{f_{g_{i}}}\right)^{2}} \\
& +E_{2} \sum_{j=1}^{P+K}\left(\sum_{i=1}^{S}\left(h_{t, q} V / a_{j}\right)-1 \mid\right) \\
t & =\left\{\begin{array}{cl}
i / c & \text { If modulus of } \mathrm{i} / \mathrm{c} \text { is zero } \\
\text { quotient of }(\mathrm{i} / \mathrm{c})+1 & \text { Otherwise }
\end{array} \quad\right. \\
q & =\left\{\begin{array}{cl}
c & \text { If modulus of } \mathrm{i} / \mathrm{c} \text { is zero } \\
\bmod \text { ulus of }(\mathrm{i} / \mathrm{c}) & \text { Otherwise }
\end{array}\right. \\
V & = \begin{cases}1 & \text { If } \mathrm{g}_{\mathrm{i}}=\mathrm{j} \\
0 & \text { Otherwise }\end{cases}
\end{aligned}
$$

In Eq. (10), $h_{t, q}$ is the demand at cell $l_{t, q} \cdot r_{t}$ and $c_{q}$ indicate the location of demand cell $l_{\mathrm{t}, \mathrm{q}}$. $\mathrm{a}_{\mathrm{j}}$ is the capacity of facility $\mathrm{j}$. $\mathrm{g}_{\mathrm{i}}$ is the value of each gene of chromosome of external GA, which indicates the facility center i. $g_{i}$ can take a number from 1 to $\mathrm{P}+\mathrm{K}$. $\mathrm{f}_{\mathrm{g}}$ is the location of facility $\mathrm{g}_{\mathrm{i}}$. Constant c in Eq. (11) and (12) is the number of columns cell. $\mathrm{E}_{1}$ and $\mathrm{E}_{2}$ are constants.

Objective function consists of two parts. The first part calculates the sum of weighted Euclidean distances between demand cells and facility centers. The second part of Eq. (10) controls the amount of demands that is referred to each facility center. If the sum of the referred demands to one center is not close to capacity of that facility center, the value of OF is increased. Therefore, this part controls the capacities. To join these two parts, we used $E_{1}$ and $E_{2}$ as weighing parameters.

\subsubsection{External GA}

External GA is the one that is applied to choose the best $\mathrm{P}$ location for facilities among the $\mathrm{M}$ potential facility places.

Each set of coordination setting is packed into a chromosome. Each chromosome consists of 2P realcoded genes, including the number of rows and column of cells, which contains the selected facility locations among the potential facility places (Fig. 3).

\begin{tabular}{|l|l|l|l|l|l|l|l|l|l|}
\hline $\mathrm{X}_{1}$ & $\mathrm{Y}_{1}$ & $\mathrm{X}_{2}$ & $\mathrm{Y}_{2}$ & & $\ldots$ & & $\mathrm{Y}_{\mathrm{p}-1}$ & $\mathrm{X}_{\mathrm{P}}$ & $\mathrm{Y}_{\mathrm{P}}$ \\
\hline
\end{tabular}

Fig. 3. The structure of a chromosome for external GA

The value of objective function for external genetic algorithm is the calculated value of Eq. (10), which is the optimum value of internal GA.

\subsubsection{The integration of two GAs}

In the proposed method, we used external GA to solve the location problem and internal GA to challenge allocation issue. Since location and allocation are not two separate problems, we must solve the two problems simultaneously. Therefore, we combined the two GA in one algorithm in such a manner that output of one is used as input to another.

In each iteration the output of external algorithm, which is the location of new facilities, is combined with existing information; the location of existing facilities, and then is used as input parameters to internal algorithm and output of internal GA is used to evaluate the effectiveness of facility locations which are selected by external algorithm. This process continues to estimate the best location with the best allocation of demand cells by existing and new facility centers.

\section{Case Study}

To assess the efficiency of the proposed method, a sample problem is designed and its results are compared with traditional method. 
We assumed the test set includes 100 cells as demand sections with different rate of demands in each section. Two existing facility centers and also 10 potential facility places were also considered. Characteristics of cells and potential facilities are given in Table 1 and Table 2.

Table 1 . The characteristics of 100 sampled regions

\begin{tabular}{|c|c|c|c|c|c|c|c|}
\hline $\begin{array}{l}\text { Region } \\
\text { ID }\end{array}$ & $\begin{array}{r}\text { Row } \\
\text { numb } \\
\text { er }\end{array}$ & $\begin{array}{l}\text { Column } \\
\text { number }\end{array}$ & $\begin{array}{c}\text { Number } \\
\text { of } \\
\text { pupils }\end{array}$ & $\begin{array}{c}\text { Region } \\
\text { ID }\end{array}$ & $\begin{array}{c}\text { Row } \\
\text { num } \\
\text { ber }\end{array}$ & $\begin{array}{c}\text { Column } \\
\text { number }\end{array}$ & $\begin{array}{c}\text { Number } \\
\text { of } \\
\text { pupils }\end{array}$ \\
\hline 1 & 1 & 1 & 79 & 51 & 6 & 1 & 65 \\
\hline 2 & 1 & 2 & 71 & 52 & 6 & 2 & 84 \\
\hline 3 & 1 & 3 & 76 & 53 & 6 & 3 & 75 \\
\hline 4 & 1 & 4 & 67 & 54 & 6 & 4 & 77 \\
\hline 5 & 1 & 5 & 72 & 55 & 6 & 5 & 67 \\
\hline 6 & 1 & 6 & 81 & 56 & 6 & 6 & 64 \\
\hline 7 & 1 & 7 & 74 & 57 & 6 & 7 & 73 \\
\hline 8 & 1 & 8 & 65 & 58 & 6 & 8 & 61 \\
\hline 9 & 1 & 9 & 64 & 59 & 6 & 9 & 63 \\
\hline 10 & 1 & 10 & 70 & 60 & 6 & 10 & 78 \\
\hline 11 & 2 & 1 & 63 & 61 & 7 & 1 & 73 \\
\hline 12 & 2 & 2 & 63 & 62 & 7 & 2 & 75 \\
\hline 13 & 2 & 3 & 74 & 63 & 7 & 3 & 81 \\
\hline 14 & 2 & 4 & 65 & 64 & 7 & 4 & 75 \\
\hline 15 & 2 & 5 & 87 & 65 & 7 & 5 & 67 \\
\hline 16 & 2 & 6 & 64 & 66 & 7 & 6 & 63 \\
\hline 17 & 2 & 7 & 74 & 67 & 7 & 7 & 61 \\
\hline 18 & 2 & 8 & 70 & 68 & 7 & 8 & 86 \\
\hline 19 & 2 & 9 & 72 & 69 & 7 & 9 & 64 \\
\hline 20 & 2 & 10 & 75 & 70 & 7 & 10 & 68 \\
\hline 21 & 3 & 1 & 68 & 71 & 8 & 1 & 66 \\
\hline 22 & 3 & 2 & 79 & 72 & 8 & 2 & 65 \\
\hline 23 & 3 & 3 & 82 & 73 & 8 & 3 & 71 \\
\hline 24 & 3 & 4 & 66 & 74 & 8 & 4 & 63 \\
\hline 25 & 3 & 5 & 67 & 75 & 8 & 5 & 75 \\
\hline 26 & 3 & 6 & 73 & 76 & 8 & 6 & 69 \\
\hline 27 & 3 & 7 & 66 & 77 & 8 & 7 & 70 \\
\hline 28 & 3 & 8 & 68 & 78 & 8 & 8 & 76 \\
\hline 29 & 3 & 9 & 74 & 79 & 8 & 9 & 65 \\
\hline 30 & 3 & 10 & 81 & 80 & 8 & 10 & 72 \\
\hline 31 & 4 & 1 & 65 & 81 & 9 & 1 & 73 \\
\hline 32 & 4 & 2 & 74 & 82 & 9 & 2 & 63 \\
\hline 33 & 4 & 3 & 73 & 83 & 9 & 3 & 60 \\
\hline 34 & 4 & 4 & 83 & 84 & 9 & 4 & 61 \\
\hline 35 & 4 & 5 & 68 & 85 & 9 & 5 & 63 \\
\hline 36 & 4 & 6 & 71 & 86 & 9 & 6 & 71 \\
\hline 37 & 4 & 7 & 73 & 87 & 9 & 7 & 62 \\
\hline 38 & 4 & 8 & 73 & 88 & 9 & 8 & 64 \\
\hline 39 & 4 & 9 & 64 & 89 & 9 & 9 & 75 \\
\hline 40 & 4 & 10 & 68 & 90 & 9 & 10 & 68 \\
\hline 41 & 5 & 1 & 84 & 91 & 10 & 1 & 81 \\
\hline 42 & 5 & 2 & 73 & 92 & 10 & 2 & 85 \\
\hline 43 & 5 & 3 & 64 & 93 & 10 & 3 & 64 \\
\hline 44 & 5 & 4 & 69 & 94 & 10 & 4 & 66 \\
\hline 45 & 5 & 5 & 87 & 95 & 10 & 5 & 69 \\
\hline 46 & 5 & 6 & 63 & 96 & 10 & 6 & 76 \\
\hline 47 & 5 & 7 & 66 & 97 & 10 & 7 & 64 \\
\hline 48 & 5 & 8 & 67 & 98 & 10 & 8 & 70 \\
\hline 49 & 5 & 9 & 69 & 99 & 10 & 9 & 78 \\
\hline 50 & 5 & 10 & 67 & 100 & 10 & 10 & 72 \\
\hline
\end{tabular}

Table 2. The location of potential places for constructing new schools

\begin{tabular}{ccc}
\hline $\begin{array}{c}\text { Potential } \\
\text { school ID }\end{array}$ & Row number & Column number \\
\hline 1 & 5 & 5 \\
2 & 8 & 3 \\
3 & 5 & 2 \\
4 & 7 & 3 \\
5 & 4 & 6 \\
6 & 2 & 9 \\
7 & 8 & 9 \\
8 & 3 & 8 \\
9 & 2 & 3 \\
10 & 9 & 3 \\
\hline
\end{tabular}

Finally, the aim is to select the best locations for two new facility centers in such a manner that the sum of weighed distances between these 4 facilities (2 existing and 2 new selected) and 100 weighed demands is minimized. The capacity of each facility is assumed as Eq. (14) in which, the $h_{i}$ is the demand of customer $i$.

$$
a_{j}=\left(\sum_{i} h_{i}\right) /(P+K)
$$

First, we applied traditional method which solves the problem in two independent steps for the existing and new facilities. At first step, the solution allocates the demands to existing facilities considering the capacity of each facility. As shown in Fig. 4-a, there would remain some demands, which were not allocated to any existing centers due to the capacity limitation of existing facility centers. Take notice that, in the figure red and green circles indicate existing. Each small square indicates a demand region that is allocated to the same colored facility center. In the second step, the location of new facilities should be determined according to the remaining regions which had got more demands (black and blue regions in Fig. 4-b). White squares indicate the demands that have inappropriate services from any facility centers so they have to get the services from distant centers. According to remaining capacity of new centers the result is as shown in Fig. 4-c.

Now we want to solve the same problem by our proposed solution. For this problem we considered the maximum generation for a run of the internal GA 1000 and for external GA 200. The initial populations for both internal and external GAs were assumed 50. The result of simulation is depicted in Fig. 5.

At the end, by comparing these two figures, it is clear that, allocation for existing facilities, red and green 
squares, in Fig. 4-c is better than the Fig.5, but the total allocation in the proposed algorithm is much better than the old one, since it has the minimum objective function considering the allocation of all demands.
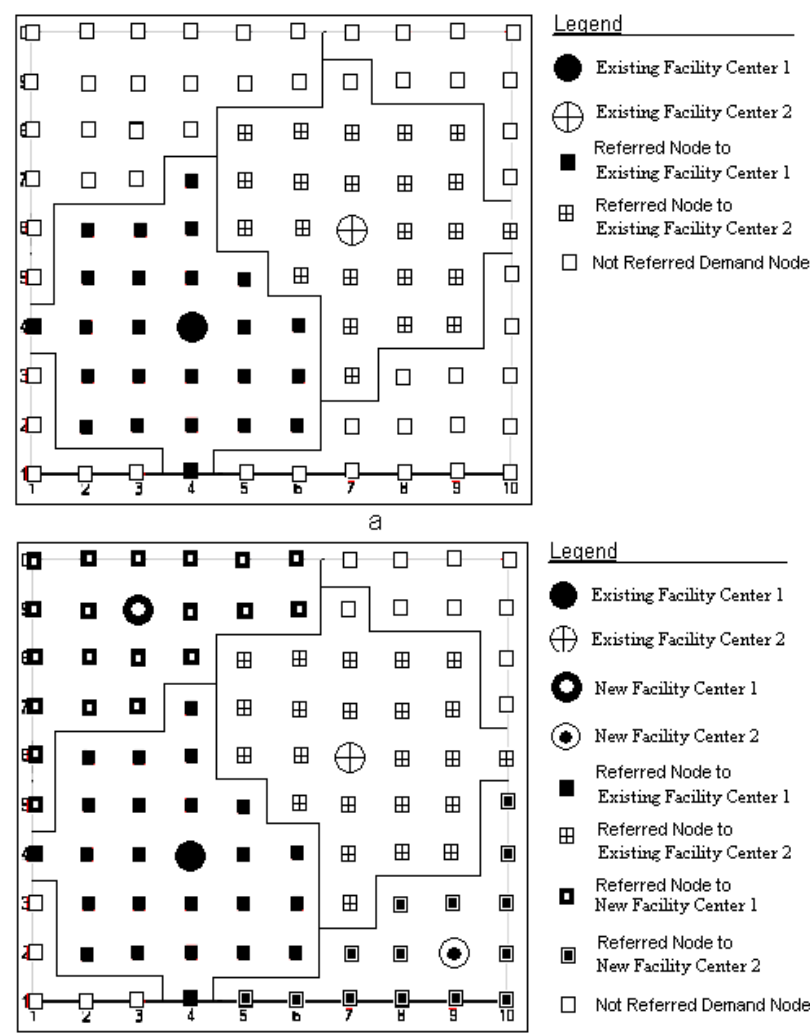

$\oplus$ Existing Facility Center 2

6 New Facility Center 1

(-) New Facility Center 2 Referred Node to

- Referred Node to

由 Referred Node to Existing Facility Center 2

Referred Node to

New Facility Center

Referred Node to

$\square$ Not Referred Demand Node

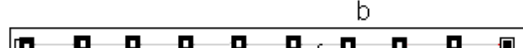

Legend

Existing Facility Center 1

$\oplus$ Existing Facility Center 2

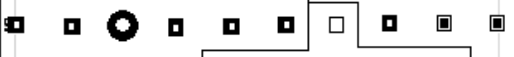

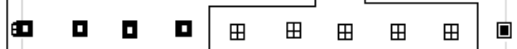

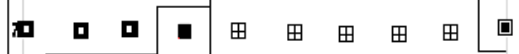

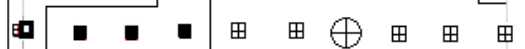

New Facility Center 1

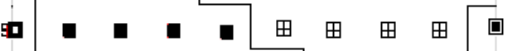

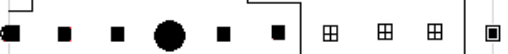

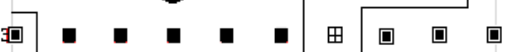

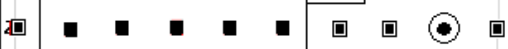

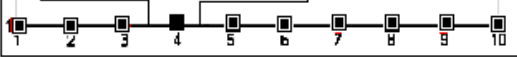

- Hew Facility Center 2

Referred Node to

Existing Facility Center 1

田 Referred Node to

Existing Facility Center 2

Referred Node to

New Facility Center

Referred Node to New Facility Center 2

Fig. 4. Steps of traditional method

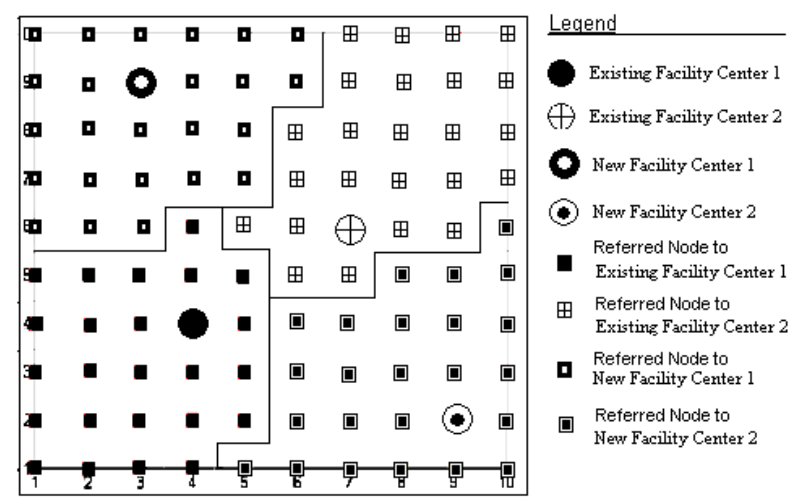

Fig. 5. The result of proposed method

The numerical comparison of two methods is given in table 3.

Table 3. Comparing the results of two methods

\begin{tabular}{|c|c|c|c|}
\hline & $\begin{array}{l}\text { Sum of } \\
\text { the weighted } \\
\text { distances } \\
\text { between } \\
\text { existing } \\
\text { facility centers } \\
\text { and their } \\
\text { referred } \\
\text { demands }\end{array}$ & $\begin{array}{l}\text { Sum of } \\
\text { the weighted } \\
\text { distances } \\
\text { between new } \\
\text { facility } \\
\text { centers and } \\
\text { their referred } \\
\text { demands }\end{array}$ & $\begin{array}{c}\text { Sum of the } \\
\text { weighted } \\
\text { distances } \\
\text { between all } \\
\text { facility } \\
\text { centers and } \\
\text { their } \\
\text { referred } \\
\text { demands }\end{array}$ \\
\hline $\begin{array}{c}\text { Traditional } \\
\text { method }\end{array}$ & 6830.2 & 11275.3 & 18105.5 \\
\hline $\begin{array}{c}\text { Proposed } \\
\text { method }\end{array}$ & 8189.4 & 6940.6 & 15130.0 \\
\hline
\end{tabular}

\section{Conclusions}

In this paper, the capacitated multi-source Weber problem is studied. We consider an extended facility location problem that involves the decision of facility locations and service allocations simultaneously. A case study was implemented to show that the proposed method is feasible for solving capacitated multi-source Weber problem.

In this problem, we considered that some service centers exist and new centers should be located to reach the best allocation of demands by all existing and new centers. The method is appropriate for solving the problems in which we can change the existing allocation to obtain new needful sub regions to establish centers.

In our proposed method, location and allocation problems are solved by two genetic algorithms. These 
two algorithms are applied as internal and external loops.

\section{References}

1. D. A. Schilling, Dynamic location modeling for public-sector facilities: A multicriteria approach, Decision Sci. 11(4) (1980) 714-724.

2. A. A. Alesheikh, M. J. Soltani, N. Nouri, and M. Khalizadeh, Land Assessment for Flood Spreading Site Selection Using Geospatial Information System, International J. of Environmental Sci. and Tech. 5(4) (2008) 455-462.

3. M. Bischoff and K. Klamroth, Two branch and bound methods for a generalized class of location-allocation problems, in Technical Report No. 313, In stitute of Applied Mathematics (University of Erlangen,Numberg, 2008).

4. J. Current, H. Min and D. Schilling, Multiobjective analysis of facility location decisions, European J. of Operational Research 49 (1990) 295-307.

5. A. Weber, Alfred Weber's Theory of the Location of Industries (University of Chicago, 1929).

6. S. H. Owen and M. S. Daskin, Strategic facility location: A review, European J. of Operational Research 111 (1998) 423-447.

7. M. Gen and R. Cheng, Genetic algorithms and engineering design (Wiley, New York, 1997).

8. M. Gen and R. Cheng, Genetic algorithms and engineering optimization (Wiley, New York, 2000).

9. M. A. Badri, Combining the analytic hierarchy process and goal programming for global facility location-allocation problem, International J. of Production Economics 62 (1999) 237-248.

10. M. Hodey, E. Melachrinoudis and X. Wu, Dynamic expansion and location of an airport: A multiple objective approach, Transportation Research Part A-Policy and Practice 31 (1997) 403-417.

11. I. Bongartz, P. H. Calamai and A. R. Conn, A projection method for norm location-allocation problems, Mathematical programming 66 (1994) 283-312.

12. T. Hale and C. Moberg, Location science research: a review, Annals of operations research 123 (2003) 21-35.

13. N. Megiddo and K. J. Supowit, on the complexity of some common geometric location problems, SIAM J. on Computing 13 (1984) 182-196.

14. P. Hansen, N. MladenoviLc and E. Taillard, Heuristic solution of the multisource Weber problem as a p-median problem, Operations Research Letters 22 (1998) 55-62.

15. C.R. Houck, J.A. Joines and MG. Kay, Comparison of genetic algorithms, random restart and two-opt switching for solving large location-allocation problems problem, European J. of Operational Research 20 (1996) 387-96.

16. J. Brimberg, P. Hansen, N. Mladenovic and E. D. Taillard, Improvements and comparison of heuristics for solving the uncapacitated multisource Weber problem, Operations Research 48 (2000) 444-60.

17. M. D. H. Gamal and S. Salhi, Constructive heuristics for the uncapacitated location-allocation problem, J. Of the Operational Research Society 51 (2001) 1233-40.
18. S. Salhi and M. D. H. Gamal, A GA based heuristic for the uncapacitated continuous location-allocation problem, Annals of Operations Research 123 (2003) 203-22.

19. M. Bischoff and K. Dachert, Allocation search methods for a generalized class of location-allocation problems, European J. of Operational Research 192 (2009) 793-807.

20. L. Cooper, The transportation-location problem, Operations Research 20 (1972) 94-108.

21. H. D. Sherali and T. P. Rizzo, Unbalanced capacitated pmedian problems on a chain graph with a continuum of link demands, Networks 21(2) (1991) 133-163.

22. Z. M. Zainuddin and S. Salhi, A perturbation-based heuristic for the capacitated multisource Weber problem, European Journal of Operational Research 179 (2007) 1194-1207.

23. M. Luis, S. Salhi and G. Nagy, Region-rejection based heuristics for the capacitated multi-source Weber problem, Computers \& Operations Research 36 (2009) 2007-2017.

24. S. H. Doong, C. C. Lai and C. H. Wu, Genetic subgradient method for solving location-allocation problems, Applied Soft Computing 7 (2007) 373-386.

25. C. Kahraman, O. Engin, I. Kaya and M. Yilmaz, An application of effective genetic algorithms for solving hybrid Flow Shop scheduling problems, International Journal of Computational Intelligence Systems 1(2) (2008) 134-147.

26. C. C. Lai, A novel clustering approach using hierarchical genetic algorithms, Intel. Automation Soft Computing 11 (2005) 143-153.

27. D. Beasley, D. R. Bull and R. R. Martin, An overview of genetic algorithms, Univ. computing 15 (1993) 170-181. 